\title{
Determination of phase space region for cross-check validation of the neutron detection in the BINA experiments
}

B. Włoch ${ }^{1 \star}$, K. Bodek ${ }^{2}$, I Ciepał ${ }^{1}$, M. Eslami-Kalantari ${ }^{3}$, J. Golak ${ }^{2}$, N. Kalantar-Nayestanaki ${ }^{3}$, G. Khatri ${ }^{4}$, St. Kistryn ${ }^{2}$, B. Kłos ${ }^{5}$, A. Kozela ${ }^{1}$, J. Kuboś ${ }^{1}$, P. Kulessa ${ }^{6}$, A. Łobejko ${ }^{5}$, A. Magiera ${ }^{2}$, H. Mardanpour ${ }^{3}$, J. Messchendorp ${ }^{3}$, I. Mazumdar ${ }^{7}$, W. Parol ${ }^{1}$, A.

Ramazani-Moghaddam-Arani ${ }^{3}$, D. Rozpędzik ${ }^{2}$, R. Skibiński ${ }^{2}$, I. Skwira-Chalot ${ }^{8}$, E. Stephan ${ }^{5}$, A. Wilczek ${ }^{5}$, H. Witała ${ }^{2}$, A Wrońska ${ }^{2}$ and J. Zejma ${ }^{2}$

1 Institute of Nuclear Physics Polish Academy of Sciences, PL-31342 Krakow, Poland 2 M. Smoluchowski Institute of Physics, Jagiellonian University, PL-30348 Krakow, Poland

3 KVI-CART, University of Groningen, NL-9747 Groningen, The Netherlands 4 CERN, CH-1211 Geneva, Switzerland

5 Institute of Physics, University of Silesia, PL-41500 Chorzów, Poland

6 Forschungszentrum Jülich, Institut für Kernphysik, D-52428 Jülich, Germany

7 Tata Institute of Fundamental Research, 400005 Mumbai, India

8 Faculty of Physics University of Warsaw, PL-02093 Warsaw, Poland

* boguslaw.wloch@ifj.edu.pl

November 7, 2019

Proceedings for the 24th edition of European Few Body Conference, Surrey, UK, 2-4 September 2019

\begin{abstract}
Deuteron breakup reactions are basic laboratories for testing nuclear force models. Recent improvements in the data analysis allow for direct identification of neutrons in the BINA detection setup. This opens up the opportunity to study new aspects of few-nucleon system dynamics like charge dependence of nuclear force or Coulomb interaction. In this paper we determine regions along the kinematical curves where differential cross section of deuteron-proton breakup reactions can be measured by the proton-neutron and proton-proton coincidences simultaneously. This is particularly useful for validation of the neutron detection technique.
\end{abstract}

\section{Introduction}

Investigations of few-nucleon systems gives suitable testing ground for modern nuclear interaction models. The simplest system, in which different theoretical models can be tested, are those composed of three nucleons ( $3 \mathrm{~N})$. The differential cross sections of the deuteron breakup reaction are very sensitive to various aspects of the system dynamics, not only to the effects of the three nucleon force (3NF), but also to the long-range Coulomb interaction and to its interplay with strong forces. Exact solutions of the Faddeev equations with any modern nuclear potentials like the CD Bonn [1], Argonne V18 [2] or Nijmegen I and II [3] combined with models of 3NF like the Tucson-Melbourne [4] or the Urbana IX [5] are available. There are 
also alternative methods of construction of the potentials like coupled-channel approach [6] and, most importantly, the ones based on Chiral Effective Field Theory [7].

A new generation experiments devoted to studies of few-nucleon systems were carried out at KVI in Groningen (the Netherlands) with the use of the BINA detector and with the deuteron beam provided by the AGOR cyclotron [8]. A large set of high precision data of ${ }^{2} \mathrm{H}(d, d p) n$ [9] and ${ }^{1} \mathrm{H}(d, p p) n$ [10] deuteron breakup reaction were obtained at a beam energy of $160 \mathrm{MeV}$. The experiment was a continuation of previous very successful studies $[11,12]$. In this analysis we are focused on two kinds of the deuteron-proton breakup reactions, one with the detection of two protons and the other with proton and neutron detected in the BINA.

\section{Experimental Setup}

The BINA detection system is characterized by a large angular acceptance. It has been specially designed for investigations of few-nucleon systems in the intermediate energy range. It is composed of two main parts, the Forward Wall covering polar angles from $16^{\circ}$ to $40^{\circ}$ and the Backward Ball covering range from $40^{\circ}$ to $165^{\circ}$. The Backward Ball consists of 149 triangular phoswich detectors arranged in a fullerene-like shape. It also plays the role of a vacuum-tight scattering chamber with a liquid deuterium or hydrogen target inside. The Forward Wall is composed of a multi-wire proportional chamber (MWPC), 12 vertical strips made of thin plastic scintillator stripes $(\Delta E)$ and 10 long and thick horizontally arranged, plastic scintillator slabs forming a stopping detector $(E)$. The vertical and the horizontal scintillators form an array of virtual $\Delta E$ - $E$ telescopes used for particle identification. Detailed information about the detector can be found e.g.in $[13,14]$.
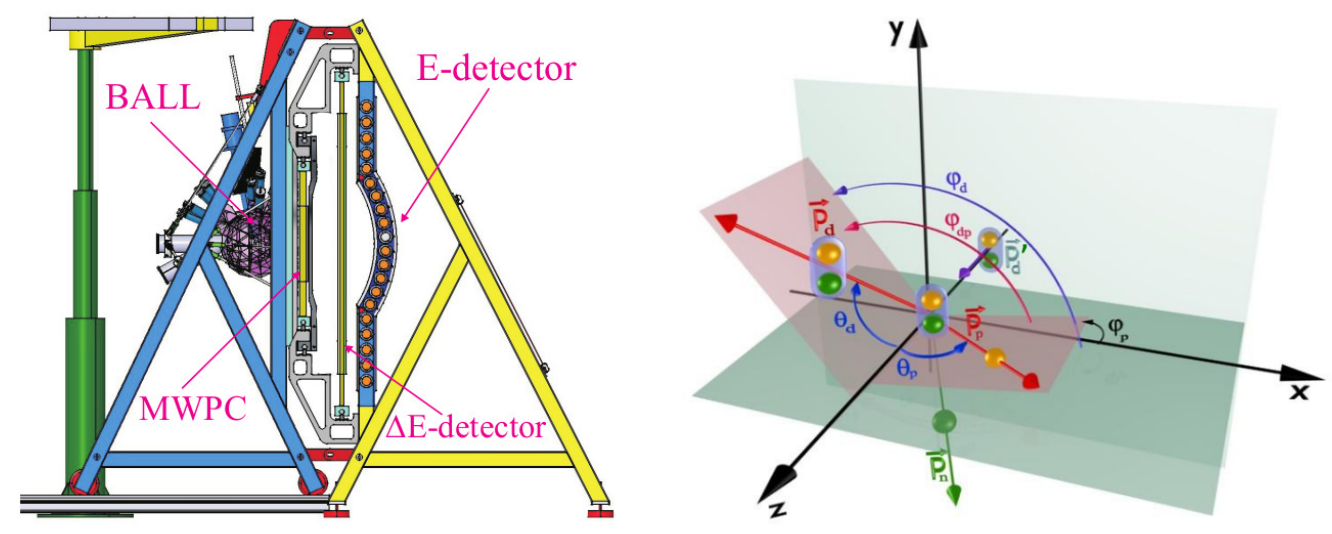

Figure 1: Left panel: A side view of the BINA experimental setup. Right panel: A schematic view of the breakup reaction with indicated polar $\left(\theta_{p}\right)$, azimuthal $\left(\phi_{p}\right)$ and the relative azimuthal $\left(\Delta \phi_{p p}\right)$ angles of the two detected particles in the laboratory reference frame. Figures adopted from [15].

\section{Neutron detection}

For the neutron detection we use the Forward Wall. Among all the elements of the BINA setup, the thick $E$ scintillators have the highest efficiency for the neutron detection, estimated at 
around $10 \%[16,17]$ which is consistent with the other experiments with a similar detection geometry and scintillating material [18]. In our detection technique, the $\Delta \mathrm{E}$ detector and MWPC work as an active veto, rejecting possible events with charged particles except one proton necessary to identify the reaction. Signals in each thick E bar were read out from both ends by photomultipliers, allowing to obtain the information on the position.

The position of the detected neutron along the horizontal (" $x ")$ axis can be determined with two different methods. A TDC method is based on the time asymmetry between left and right photomultipliers while an ADC method uses the difference in the pulse height. Both methods provide independent information on the particle position and combined together result in position reconstruction with resolution of $\sigma_{x}=11.6 \mathrm{~mm}$ (see Fig. 2). Unfortunately, these methods cannot be used for a position reconstruction along the vertical axis. Instead, we use the granularity of the $E$ detector. The geometrical center of the scintillator gives a rough position in the $y$-axis with a maximal error of about $40 \mathrm{~mm}$.

Taking the sum of the left and the right TDC signals removes the dependence on the hit position and allows to calculate time of flight (TOF) for a given particle. Using this information, the energy of the particle can be directly calculated. Fig. 2 illustrates the resolution of the energy reconstruction for protons with the use of TOF. To find TOF of a neutron, at least one accompanying charged particle must be registered in the Wall detector in order to calculate the time $\mathrm{T}_{0}$ of the reaction. Having the neutron position and energy reconstructed, one can calculate its momentum.
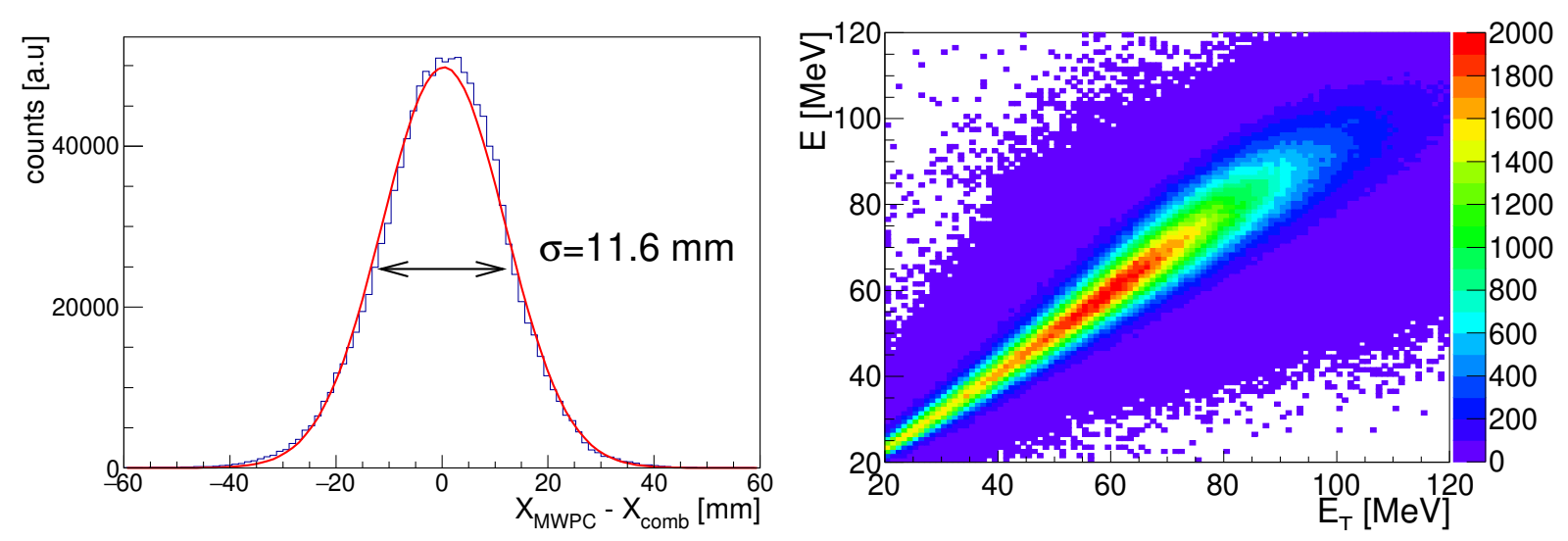

Figure 2: Check of momentum reconstruction methods on the basis of registered protons: Left panel: Histogram of the differences between the $x$-coordinate reconstructed from combined TDC and ADC method and the $x$-coordinate obtained from MWPC. Right panel: Energy reconstructed from the deposited energy in the $E$ detector versus energy obtained from the time of flight method $E_{T}[19]$.

\section{Proton-neutron cross-check regions}

A commonly used representation of experimental data in three-body breakup reaction is a 5fold differential cross section. The definition of angles is shown in Fig. 1. The three particles in the final state can be described with 9 variables. The energy and momentum conservation laws and the axial symmetry of the reaction reduces this number to 5 variables, e.g., $E_{p 1}, E_{p 2}$, 
$\theta_{p 1}, \theta_{p 2}, \Delta \phi=\phi_{p 1}-\phi_{p 2}$. The $E_{p 1}$ and $E_{p 2}$ are related by the formula:

$$
E_{p 1}+E_{p 2}-\sqrt{2 E_{\text {beam }} E_{p 1}} \cos \theta_{p 1}-\sqrt{2 E_{\text {beam }} E_{p 2}} \cos \theta_{p 2}+\sqrt{E_{p 1} E_{p 2}} \cos \theta_{p 1 p 2}=\frac{Q-E_{\text {beam }}}{2}
$$

where: $\cos \theta_{p 1 p 2}=\cos \theta_{p 1} \cos \theta_{p 2}+\sin \theta_{p 1} \sin \theta_{p 2} \cos \left(\phi_{p 1}-\phi_{p 2}\right), E_{\text {beam }}$ is the beam (deuteron) energy, $E_{p 1}, \theta_{p 1}$ are the energy and polar angle of the first proton, $E_{p 2}, \theta_{p 2}$ are the energy and polar angle of the second proton and $Q$ is the binding energy of the deuteron.

For a chosen angular configuration $\left(\theta_{p 1}, \theta_{p 2}, \Delta \phi\right)$ the relation between energy of the two particles defines a so-called "kinematical curve" or kinematics. Examples of bunch of kinematics are presented in Fig. 3. In order to present the cross section distributions and to avoid the confusion about which particle is the "first" and which is the "second" one, one defines the $S$-variable, an arc-length along the kinematics. It is clear that complete breakup kinematics can be reconstructed based on the knowledge of three momentum components of one particle and emission angles of the second particle.

To determine the regions of interest where both kinematics of ${ }^{1} \mathrm{H}(d, p p) n$ and ${ }^{1} \mathrm{H}(d, p n) p$ overlap, the following procedure have been developed based on theoretical cross sections. A set of theoretical cross sections based on the CDBonn potential [20] for both mentioned reactions has been calculated. The energy thresholds for a detection of protons and neutrons in the BINA setup have been introduced. For each analyzed proton-proton angular configuration, the energy and emission angles of the corresponding neutron were calculated for every point $\left(E_{p 1}, E_{p 2}\right)$ on the proton-proton kinematics. If the calculated neutron angles are within the acceptance of the detector (Wall) and its energy exceed the detection threshold, then such a point is considered as valid for the cross-check analysis. For each such point, $\left(E_{n}, E_{p}\right)$, the position on the proton-neutron kinematics was determined. Having this regions identified, one can calculate the corresponding values of the cross sections for ${ }^{1} \mathrm{H}(d, p p) n$ and ${ }^{1} \mathrm{H}(d, p n) p$ reactions, respectively. Examples of the obtained distributions are shown in Fig. 4. This procedure allows for direct cross-check the analysis based on the proton-proton and protonneutron coincidences.
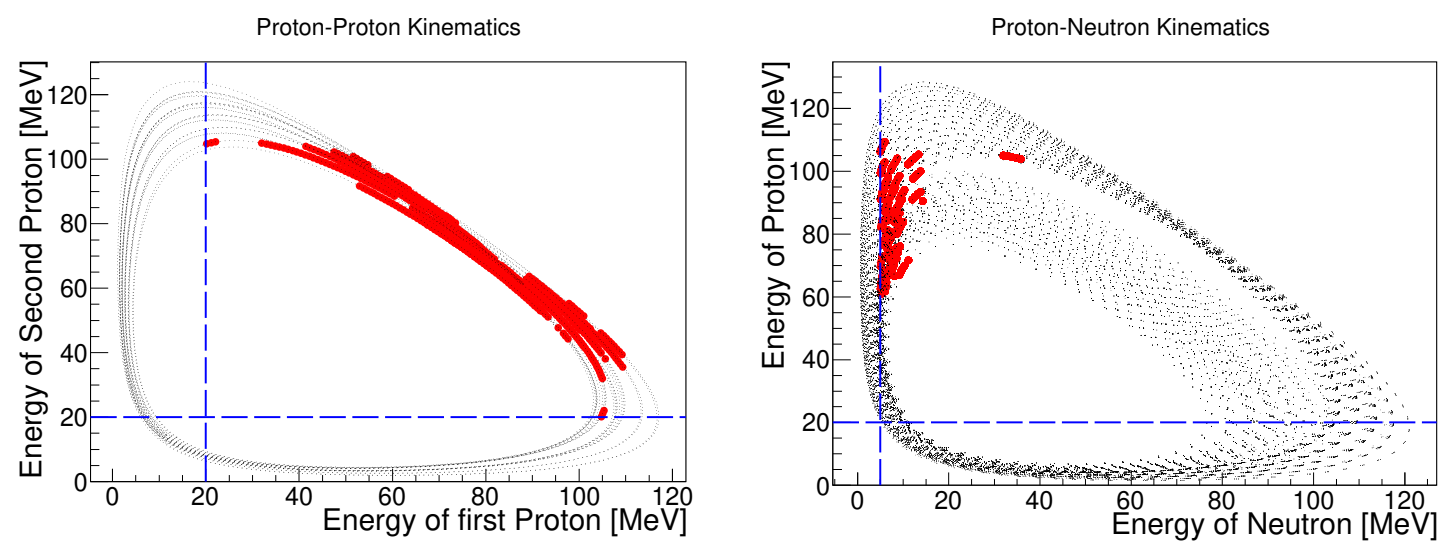

Figure 3: Left panel: A set of proton-proton kinematics for various combinations of $\theta_{p 1}, \theta_{p 2}$ and $\Delta \phi$ angles. Red lines represent the regions, where the corresponding neutron can be detected in the BINA setup together with the proton-proton coincidences. Blue dashed lines refer to applied detection thresholds. Right panel: The same regions as in the left panel but for the neutron-proton kinematics. 

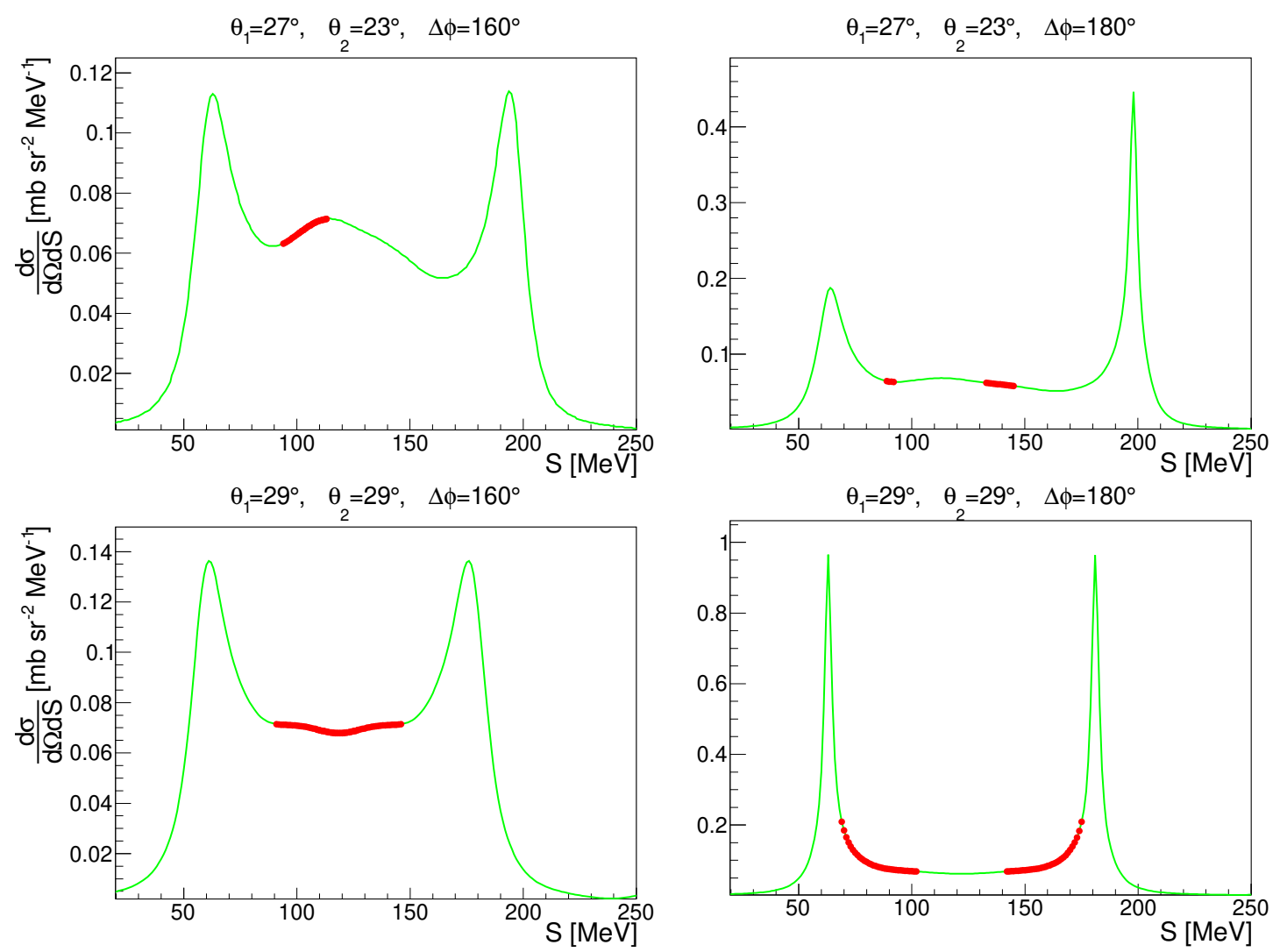

Figure 4: Examples of the differential cross sections of ${ }^{1} \mathrm{H}(d, p p) n$ breakup for two symmetric and two asymmetric angular configurations, specified in the figure. Green lines refers to the theoretical calculations based on the CD Bonn potential. Red solid lines and dots represent the regions useful for the cross-check analysis.

\section{Conclusion}

The newly developed neutron detection method is very promising. In order to check our detection method, a simple analysis of kinematic relationship of deuteron-proton breakup reaction has been performed. The phase-space regions characterized by the possibility to extract cross section independently from neutron-proton and proton-proton coincidences has been found. The analysis allowed to select 178 experimental points that can be used for a cross-check within the acceptance of our detection setup. In the next step, this procedure will be applied for the data. By comparing the final cross section calculated from neutron-proton and corresponding proton-proton coincidences one will be able to study systematic uncertainties of most important tools used for analysis involving neutrons (detection efficiency, configurational efficiency, energy reconstruction etc.). The results will be used in further studies of the deuteron breakup reaction.

\section{Acknowledgements}

The numerical calculations were partially performed on the supercomputer cluster of the JSC, Jülich, Germany. 
Funding information This work was supported by the Polish National Science Center under Grants No. 2012/05/E/ST2/02313, No. 2016/22/M/ST2/00173 and No. 2016/21/D/ST2/01173.

\section{References}

[1] R. Machleidt, F. Sammarruca and Y. Song, Nonlocal nature of the nuclear force and its impact on nuclear structure, Phys. Rev. C, 53, R1483, (1996), doi:10.1103/PhysRevC.53.R1483.

[2] R. B. Wiringa, V. G. J. Stoks and R. Schiavilla, Accurate nucleon-nucleon potential with charge-independence breaking, Phys. Rev. C, 51, 38, (1995), doi:10.1103/PhysRevC.51.38.

[3] V. G. J. Stoks, et al., Construction of high-quality NN potential models, Phys. Rev. C, 49, 2950, (1994), doi:10.1103/PhysRevC.49.2950.

[4] S. A. Coon and W. Glöckle, Two-pion-exchange three-nucleon potential: Partial wave analysis in momentum space, Phys. Rev. C 23, 1790, (1981), doi:10.1103/PhysRevC.23.1790.

[5] B. S. Pudliner, et al., Quantum Monte Carlo calculations of nuclei with A < 7, Phys. Rev. C, 56, 1720, (1997), doi:10.1103/PhysRevC.56.1720.

[6] A. Deltuva, R. Machleidt and P. Sauer, Realistic two-baryon potential coupling two-nucleon and nucleon- $\Delta$-isobar states: fit and applications to three-nucleon system, Phys. Rev. C, 68, 024005, (2003), doi:https://doi.org/10.1103/PhysRevC.68.024005.

[7] E. Epelbaum, et al., Three-nucleon forces from chiral effective field theory, Phys. Rev. C, 66, 1, (2002), doi:10.1103/PhysRevC.66.064001.

[8] A. Ramazani-Moghaddam-Arani, et al., Spin observables in the three-body break-up process near the quasi-free limit in deuteron-deuteron scattering, Phys. Lett. B, 725, 282, (2013), doi:10.1016/j.physletb.2013.07.041.

[9] I. Ciepał, et al., Three-body breakup in deuteron-deuteron collisions at 160 $\mathrm{MeV}$ including quasifree scattering, Phys. Rev. C, 100, 024003, (2019), doi:10.1103/PhysRevC.100.024003.

[10] W. Parol, Investigation of three-nucleon force effects in the reaction of deuteron proton breakup at the energy of $80 \mathrm{MeV} /$ nucleon, Ph.D Thesis, Jagiellonian University, Kraków, (2015), https://fais.uj.edu.pl/documents/41628/ 761d2b13-faa4-4960-bfe9-96157aa48bd6.

[11] St. Kistryn, et al., Evidence of the Coulomb-force effects in the cross-sectionsof the deuteron-proton breakup at $130 \mathrm{MeV}$, Phys. Lett. B, 23, 641, (2006), doi:10.1016/j.physletb.2006.08.013.

[12] E. Stephan, et al., Vector and tensor analyzing powers in deuteron-proton breakup at 130 MeV, Phys. Rev. C, 82, 014003, (2010), doi:10.1103/PhysRevC.82.014003.

[13] E. Stephan, et al., THREE-NUCLEON INTERACTION DYNAMICS STUDIED VIA THE DEUTERON-PROTON BREAKUP, Int. J. Mod. Phys. A, 24, (2009), doi:10.1142/S0217751X09043973. 
[14] H. Mardanpour-Mollalar, "Investigation of nuclear forces in $d+p$ elastic and $p+d$ break-up reactions at intermediate energies", Ph.D Thesis, RUG, Groningen (2008).

[15] G. Khatri, Investigation of Deuteron Disintegration, Ph.D Thesis, Jagiellonian University, Kraków (2015), https://fais.uj.edu.pl/documents/41628/ 32ab18f5-a619-476b-b754-c02d263c0d1b.

[16] J. Kuboś, Wyznaczenie wydajnosci detektora BINA na rejestracje neutronow $w$ reakcji breakupu dp przy energii $160 \mathrm{MeV}$, BSc thesis, AGH, Kraków, (2014).

[17] M. Volkerts, A comprehensive study of proton-deuteron bremsstrahlung, Ph.D Thesis, RUG, Groningen, (2001).

[18] E. Clyde, et al., Detection Efficiency of Plastic Scintillator for Neutron Energies 4 to $76 \mathrm{Mev}$, Rev. of Sci. Instr., 33, 526, (1962), doi:10.1063/1.1717908.

[19] B. Włoch, et al., Reconstruction of the Neutron Momentum in the Deuteron Breakup Reaction, Acta Phys. Pol. B, 49, (2018), doi:10.5506/APhysPolB.49.445.

[20] H. Witała, J. Golak, R. Skibiński, Selectivity of the nucleon-induced deuteron breakup and relativistic effects, Phys. Lett. B, 634, 374, (2006), doi:10.1016/j.physletb.2006.01.070. 